\title{
Mycobacterium avium complex infection: phenotypes and outcomes
}

\author{
Michael R. Loebinger \\ Affiliation: Royal Brompton Hospital and Imperial College London, London, UK.
}

Correspondence: Michael R. Loebinger, Royal Brompton Hospital, Sydney St, London SW3 6NP, UK. E-mail: m.loebingerarbht.nhs.uk

@ERSpublications

The outcome of infection by Mycobacterium avium complex reflects clinical phenotypes http://ow.ly/sV8z30erYON

Cite this article as: Loebinger MR. Mycobacterium avium complex infection: phenotypes and outcomes. Eur Respir J 2017; 50: 1701380 [https://doi.org/10.1183/13993003.01380-2017].

\begin{abstract}
Nontuberculous mycobacteria (NTM) pulmonary disease is becoming increasingly recognised as an emerging healthcare issue. Multiple studies have demonstrated an increasing prevalence throughout the world, with evidence of significant associated morbidity, mortality and healthcare costs $[1,2]$. The most prevalent and important group leading to pulmonary disease is the Mycobacterium avium complex (MAC), which includes the Mycobacterium avium, Mycobacterium intracellulare and Mycobacterium chimaera species. These organisms are ubiquitous in the environment and, unlike Mycobacterium tuberculosis, isolation of NTM from respiratory samples does not necessarily equate to pulmonary disease. Furthermore, even radiological evidence of pulmonary disease does not always necessitate treatment [3]. The judicious use of treatment for pulmonary NTM disease is related, in part, to the long ( $>1$ year) courses of multiple antibiotics needed for treatment. In addition to problems with tolerance of the antibiotic regimens, treatment failure is common. There has been a wide range (13-86\%) of reported treatment success rates in the literature for pulmonary NTM disease $[4,5]$, influenced by disease severity, treatment regimen, microbial resistance and definition of success. A recent meta-analysis of 16 studies involving 1462 patients on macrolide containing regimes reported a treatment success rate, defined by sustained culture conversion, of $60 \%$ [6].
\end{abstract}

Historically, two radiological patterns of MAC disease have been described, with a fibrocavitary (FC) form, associated predominantly with men with chronic lung disease, and a nodular bronchiectatic (NB) form with better outcomes, more often associated with post-menopausal women. Importantly, international guidelines suggest slightly different broad treatment regimens for these types, with intermittent oral therapy often suitable for NB MAC, and daily treatment, with consideration of additional, parenteral aminoglycoside, for the FC type [7]. However, in real-world medicine, patients often do not fit nicely into these two categories. The study by $\mathrm{KoH}$ et al. [8] in this issue of the European Respiratory Journal recognises this and separates the NB type into those with (C-NB) or without (NC-NB) additional small cavities within nodules. The authors recognised that, despite this additional classification, some groups (e.g. those with predominantly FC disease in addition to some bronchiectasis) will still be disenfranchised and they termed these "unclassifiable" in the study. As demonstrated in multiple previous studies $[9,10]$, this new study also demonstrates that cavitation is associated with a worse outcome, with other risk factors such as male sex also confirming the results of previous studies. This study, however, goes further in formally categorising those with small cavities and a predominantly NB pattern, and demonstrating that

Received: July 102017 | Accepted: July 102017

Conflict of interest: Disclosures can be found alongside this article at erj.ersjournals.com

Copyright @ERS 2017 
this group (C-NB) had outcomes more similar to the FC subgroup than the NB subgroup. This level of clarity is not available from previous studies. The inference from this is that the C-NB subgroup should possibly be treated more aggressively and like the FC group, with daily therapy and consideration of parenteral aminoglycoside. Despite the statistical disparity between the groups, the success rates in all patients were high in comparison to the available literature, with $88 \%$ success in the NC-NB group, $78 \%$ in the C-NB group and $76 \%$ in the FC group. There are several important possible reasons for this. Firstly, the rate of use of parenteral aminoglycoside was higher in this cohort than other studies, with a total use of $44 \%$ in the full cohort of patients (only $25 \%$ of whom had FC disease), and in $68 \%$ of those with FC and, importantly, C-NB disease. Parenteral aminoglycoside has previously been shown to lead to a significant increase in culture conversion rates $(71.2 \%$ versus $50.7 \%)$ in a randomised controlled trial (RCT) of 146 patients randomised to intramuscular streptomycin or placebo three times per week for the first 3 months of therapy [11] and this has also been supported by other cohort studies [12]. However, another explanation for the high success rates is that out of the initial cohort, 85 patients were excluded due to not completing $\geqslant 12$ months of treatment. Reasons for this lack of treatment completion may include death, intolerance and lack of perceived benefit, and if these patients are included in the denominator (as would occur with an intention-to-treat analysis), the overall success rate reduces to $71 \%$ (instead of 84\%). Furthermore, patients with "worse" disease and cavitation are likely to be overrepresented in this excluded subgroup. This, together with the lower numbers of patients in the included FC (123 patients) and C-NB (80 patients) subgroups, would lead to much lower treatment success rates than the headline figures for those with cavitation. It is also important to realise the limitations of generalising the results of a single-centre cohort. This is particularly important in NTM disease. Studies have demonstrated that the issues and respiratory disease caused by different NTM species vary significantly by location, both between and within countries, which is likely to be due to interactions between the pathogen, the host and the environment $[13,14]$.

The definition of treatment success in this study relates to sputum conversion maintained for $\geqslant 12$ months. This study also confirms the results of earlier studies, that after treatment success, a significant proportion of patients re-isolate NTM. This study described reisolation of NTM in 29\% (118 out of 402) of patients that completed treatment, which is less than the $48 \%$ in another recent cohort study of 180 patients with $\mathrm{NB}$ MAC, but is still highly significant [5]. In both studies, in the majority $(\sim 75 \%)$ of cases that were tested, the new isolate was genetically nonidentical to the initially treated isolate and the regrowth was thought to relate to reinfection as opposed to relapse. This is most likely to represent a host predisposition to NTM pulmonary disease and/or a regular environmental source/contact. The presence of multiple different isolates within an individual at a point in time may also be a possibility and several studies have demonstrated several different NTM species within the same individual [15]. From a clinical point of view, the reisolation rate (whether relapse or reinfection) should be taken into account in perceived treatment success and decision to treat. It also brings into question the length of treatment regimens. These have characteristically been for 12 months following culture conversion (dated at the first negative culture); however, the present study cites the higher relapse rate in the cavitary subgroup and suggests the possibility of longer management courses in some cases. The other question is whether a long-term prophylactic course could be used to prevent reinfection.

With the increasing prevalence of NTM, there has been expanding interest from physicians and industry alike. The number of (predominantly cohort) studies has increased, a phase III multicentre study for refractory MAC has recently completed [16], and we await the imminent publication of guidelines from the British Thoracic Society, and a separate collaboration between the European Respiratory Society, American Thoracic Society, Infectious Disease Society of America, and European Society of Clinical Microbiology and Infectious Diseases. Studies such as that in this issue [8], which include large, prospective cohorts, certainly help inform best practise but, despite this, treatment success is suboptimal, and the need for RCTs and drugs to determine better regimens remains as acute as ever.

\section{References}

1 Adjemian J, Olivier KN, Seitz AE, et al. Prevalence of nontuberculous mycobacterial lung disease in U.S. Medicare beneficiaries. Am J Respir Crit Care Med 2012; 185: 881-886.

2 Diel R, Jacob J, Lampenius N, et al. Burden of non-tuberculous mycobacterial pulmonary disease in Germany. Eur Respir J 2017; 49: 1602109.

3 Hwang JA, Kim S, Jo KW, et al. Natural history of Mycobacterium avium complex lung disease in untreated patients with stable course. Eur Respir J 2017; 49: 1600537.

4 Lam PK, Griffith DE, Aksamit TR, et al. Factors related to response to intermittent treatment of Mycobacterium avium complex lung disease. Am J Respir Crit Care Med 2006; 173: 1283-1289.

5 Wallace RJ Jr, Brown-Elliott BA, McNulty S, et al. Macrolide/azalide therapy for nodular/bronchiectatic Mycobacterium avium complex lung disease. Chest 2014; 146: 276-282.

6 Kwak N, Park J, Kim E, et al. Treatment outcomes of Mycobacterium avium complex lung disease: a systematic review and meta-analysis. Clin Infect Dis 2017 [in press https://oi.org/10.1093/cid/cix517]. 
7 Griffith DE, Aksamit T, Brown-Elliott BA, et al. An official ATS/IDSA statement: diagnosis, treatment, and prevention of nontuberculous mycobacterial diseases. Am J Respir Crit Care Med 2007; 175: 367-416.

8 Koh W-J, Moon SM, Kim S-Y, et al. Outcomes of Mycobacterium avium complex lung disease based on clinical phenotype. Eur Respir J 2017; 50: 1602503.

9 Zoumot Z, Boutou AK, Gill SS, et al. Mycobacterium avium complex infection in non-cystic fibrosis bronchiectasis. Respirology 2014; 19: 714-722.

10 Hayashi M, Takayanagi N, Kanauchi T, et al. Prognostic factors of $634 \mathrm{HIV}$-negative patients with Mycobacterium avium complex lung disease. Am J Respir Crit Care Med 2012; 185: 575-583.

11 Kobashi Y, Matsushima T, Oka M. A double-blind randomized study of aminoglycoside infusion with combined therapy for pulmonary Mycobacterium avium complex disease. Respir Med 2007; 101: 130-138.

12 Ahn $\mathrm{CH}$, Ahn SS, Anderson RA, et al. A four-drug regimen for initial treatment of cavitary disease caused by Mycobacterium avium complex. Am Rev Respir Dis 1986; 134: 438-441.

13 Hoefsloot W, van Ingen J, Andrejak C, et al. The geographic diversity of nontuberculous mycobacteria isolated from pulmonary samples: an NTM-NET collaborative study. Eur Respir J 2013; 42: 1604-1613.

14 Jankovic M, Samarzija M, Sabol I, et al. Geographical distribution and clinical relevance of non-tuberculous mycobacteria in Croatia. Int J Tuberc Lung Dis 2013; 17: 836-841.

15 Cowman S, Burns K, Benson S, et al. The antimicrobial susceptibility of non-tuberculous mycobacteria. J Infect 2016; 72: 324-331.

16 Olivier KN, Griffith DE, Eagle G, et al. Randomized trial of liposomal amikacin for inhalation in nontuberculous mycobacterial lung disease. Am J Respir Crit Care Med 2017; 195: 814-823. 